\title{
La pratique du journal : outil de réflexivité et d'apprentissage en FLE
}

\section{Omar Kharbouch}

\section{(2) OpenEdition \\ 1 Journals}

Édition électronique

URL : https://journals.openedition.org/esp/4508

DOI : $10.4000 /$ esp.4508

ISSN : 2532-0319

Éditeur

Centre d'Information sur l'Éducation Bilingue et Plurilingue

\section{Édition imprimée}

Date de publication : 1 décembre 2019

Pagination : 43-48

ISSN : 1127-266X

\section{Référence électronique}

Omar Kharbouch, «La pratique du journal : outil de réflexivité et d'apprentissage en FLE », Éducation et sociétés plurilingues [En ligne], 47 | 2019, mis en ligne le 01 septembre 2021, consulté le 01 septembre 2021. URL : http://journals.openedition.org/esp/4508; DOI : https://doi.org/10.4000/esp.4508 


\section{LA PRATIQUE DUJOURNAL: OUTIL DE RÉFLEXIVITÉ ET D’APPRENTISSAGE EN FLE}

\section{Omar KHARBOUCH}

"Il diario", all'infuori della sua connotazione intimista o di mediatizzazione, è poco noto come pratica di scrittura formatrice. Cercheremo qui di affrontarlo in quanto scrittura di riflessione e di autoformazione in francese lingua straniera. Passando in rassegna le grandi figure che lo hanno proposto, evocheremo di nuovo la sua definizione per estrame in seguito, nel contesto di una sperimentazione in una scuola media in Marocco, una delle sue dimensioni essenziali: la riflessività.

Parole chiave: diario, riflessività, apprendimento, pedagogia istituzionale, francese lingua straniera

The «diary», aside from its very personal connotation, is rarely discussed as a useful tool for language learning. We broach it as a reflexive form of writing in the teaching of French as a foreign language. After a glimpse at the important figures who have promoted it, we shall discuss its definition, before developing one of its fundamental characteristics - reflexivity - based on an experiment carried out in a Moroccan high-school.

Key-zerds: diary, reflexivity, learners, pédagogie institutionnelle, French as a foreign language.

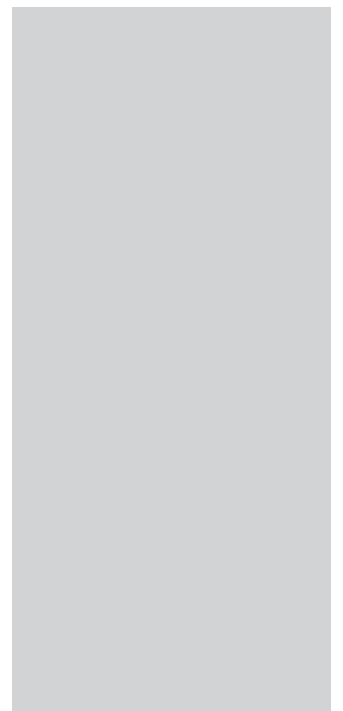

Oi J. Locke (1632-1704) et M-A. Jullien (1775-1848) ont été les précurseurs de cette pratique, c'est à J. Korczak (18781942) qu'on doit son intégration dans le monde de l'éducation, comme un outil d'apprentissage et de développement de la réflexivité mis à la disposition des apprenants. Mais, ce n'est que durant la deuxième moitié du vingtième siècle que la théorisation de cette pratique a préoccupé un grand nombre de chercheurs et praticiens institutionnalistes. On peut citer, parmi eux, R. Lourau qui a développé tout un discours sur la fonction du «hors-texte» du journal dans le contexte de la recherche et de l'écriture scientifiques. On peut aussi évoquer G. Lapassade et sa pratique du journal comme mode d'exploration ethnographique. De nos jours, une grande communauté s'inscrit dans la tradition du journal dont le foyer principal est le département des sciences de l'éducation à l'Université Paris 8 . Il s'agit d'une génération de chercheurs, dont les plus connus sont Rémi Hess et sa pratique du journal comme moyen de critique du quotidien, et $\mathrm{P}$. Ville et sa préconisation du diarisme dans les démarches socianalytiques. En somme, les membres du courant de l'Analyse et de la pédago- 


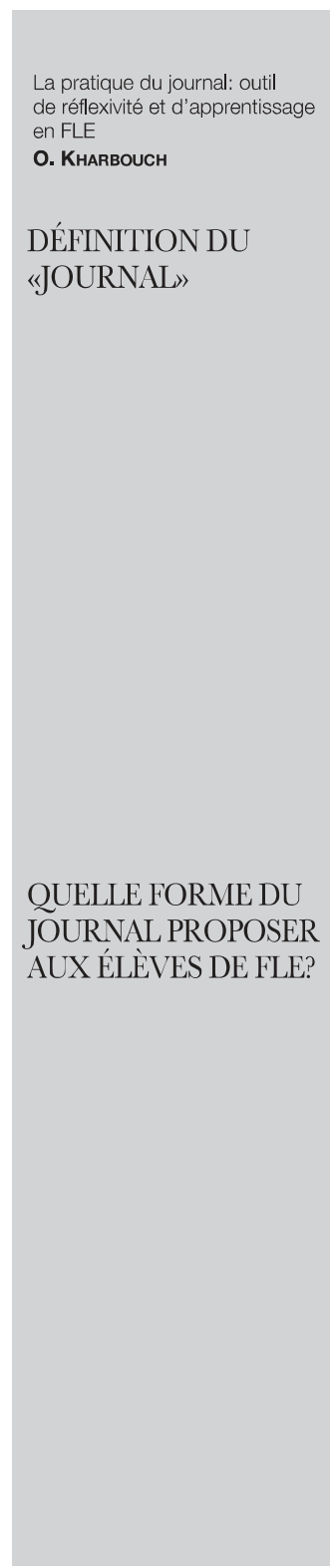

gie institutionnelles s'adonnent presque tous au diarisme.

La notion de journal, telle qu'elle est définie dans le domaine des Sciences de l'éducation, hérite des acceptions courantes quelques caractéristiques: tout comme les écrits de presse, le journal est un écrit «extime» (Hess \& Crépeau 2010), conçu pour être lu par ses pairs et, éventuellement, pour être diffusé auprès du grand public. D'autre part, à l'instar du journal intime, il relève du genre biographique: texte sans prétention littéraire accueillant une narration personnelle et progressive qui obéit à la chronologie des faits relatés et à celle de l'acte d'écrire lui-même. D'où, par ailleurs, la raison d'avoir privilégić le nom de «journal» (qui renvoic à la temporalité de l'écriture) à celui de «carnet».

L'engagement dans cette écriture permet au diariste (scripteur) de se distancer de soi, en se regardant se dé-composer et, par conséquent, elle fait émerger chez lui le plaisir de se construire et de se voir différemment (Hess 2006). Or, face au plaisir, la contrainte du temps est omniprésente; et le risque psychique de se dévoiler et d'être seul face à soi et à ses conflits peut conduire le diariste à se construire des rites non-communs. Ainsi, le journal est une écriture biographique réflexive qui sert à comprendre sa propre expérience, ses continus et discontinus et à «mettre en lumière ses dérèglements et ses confusions, voire même les provoquern (Cormery 2014).

Le groupe-objet de mon expérimentation est constitué d'une vingtaine d'élèves entre treize et seize ans. Ils étaient en $4^{\text {ème }}$ au collège et je les prenais en charge en tant qu'enseignant du FLE (au sud rural du Maroc). Dès le départ, me référant à la perspective hypothéticodéductive qui cadre ma recherche, il a fallu spécifier quelle forme de journal leur proposer, comment l'investir et, au final, dans le but de prouver quelles hypothèses.

En effet, vu l'exigence méthodologique et les attentes du système éducatif, j'ai opté, en m'inspirant de Korczak, pour le journal d'apprentissage. Celui-ci étant un dispositif/outil à l'aide duquel l'élève-diariste pourra «pointer, [ses apprentissages], ses hypothèses et ses trouvailles, à propos d'un objet» (Korczak 2006). Cette formulation intègre parfaitement le journal, en termes de valorisation et de sanction, aux autres activités dépendantes de la discipline enseignée en classe (FLE) et dont il pourrait être le support, tels que les exposés, comptes-rendus, recherches...

Après mon exposé d'initiation, n'esquissant pas une prescription magistrale et ferme, l'activité demandée aux élèves a consisté en ceci: «écrire dans un journal individuel, au jour le jour, selon les 


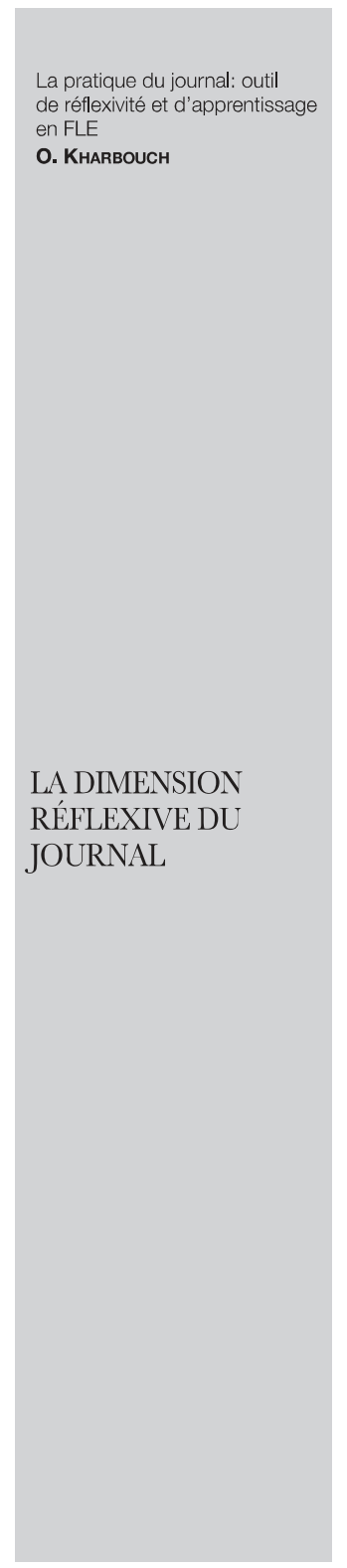

conditions de chacun, et y rapporter, à chaque entrée (une page de carnet), un ou deux faits à commenter, à apprécier ou à critiquer». Les exemples de sujets à traiter (comptes-rendus, réflexions sur un concept étudié...) leur ont été donnés à titre indicatifs tout en les appelant à libérer leur créativité et à varier leurs sujets (ressenti personnel, aspirations, projets...). Et, à la fin, la relecture do leurs écrits a été vivement sollicitée, et la lecture en classe de certains fragments sera, au côté de la tenue du journal, un objet de valorisation à comptabiliser dans la fiche de notes de chaque élève.

Ainsi, parmi les hypothèses formulées en réponse à ma problématique de recherche qui consiste à vérifier «en quoi la pratique du journal peut profiter aux élèves collégiens en classe de langue étrangère», nous focaliserons sur celle énonçant l'émergence de la dimension réflexive de cette pratique. Néanmoins, s'agissant de pratique d'apprentissage, cette dimension n'est point dissociable de celle d'outil/tiers éducatif; les deux permettant de conscientiser et capitaliser des savoirs et contribuant, ainsi, à «la formation de soi au long cours de l'existence») (Pineau 2012) et à l'épanouissement de l'élève.

On entend par «réflexivité» une démarche analytique qui a pour objet l'analyse de ses propres textes-réflexions et de ses propres méthodologies d'analyse. Il s'agit d'une métacognition par laquelle le sujet, ou en l'occurrence l'élève, se permet d'intégrer sa pratique et sa propre personne à son sujet d'étude.

Soulignons également que la simple mise en mots d'une expérience par l'écrit est une activité réflexive, qui fait du journal un espace propice à l'apprentissage autonome fondé sur la réflexivité (Little \& al 2011). Celle-ci s'exprimera par le passage de l'éprouvé au réfléchi, du vécu au conçu (Lahire 2008). Elle pourra se cristalliser, chez l'élève-diariste, en prenant la forme de retours critiques sur ses expériences passées, par des tentatives de décrire ses moments présents, ou par l'acte de se projeter dans l'avenir en proposant des solutions ou en anticipant des faits. Cette triple configuration de la réflexion (rétro-intra-prospective) amène l'ćlc̀ve à jauger ses fautes et ses implications dans le temps et à pouvoir les contrôler. Examinons donc ces aspects dans les écrits de mes élèves.

En s'interrogeant sur les causes de leur insatisfaction, leurs difficultés, leur peur de participer en classe, certains élèves ont recherché les causes de leur ressenti. Ancrés dans un questionnement du 


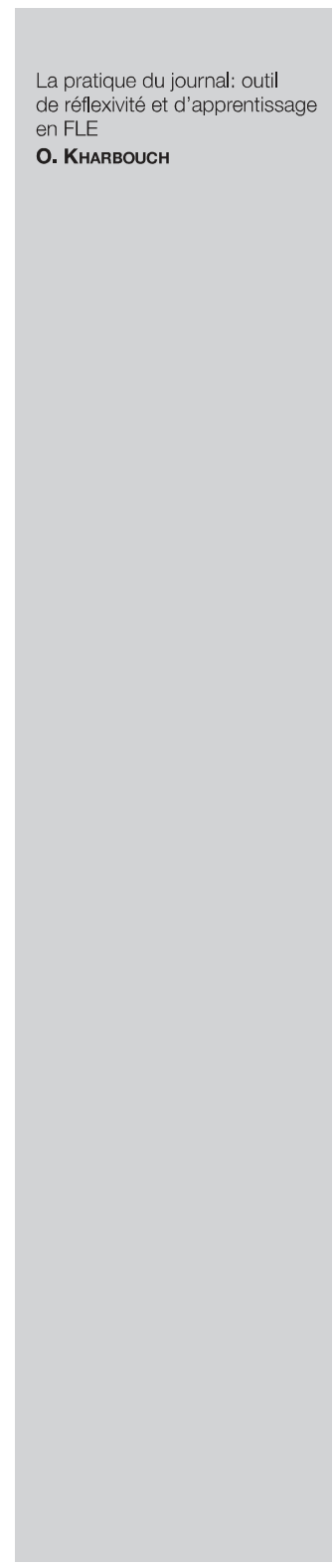

présent, leurs regards rétrospectifs leur ont permis d'expliquer même subjectivement - leur malaise d'apprenant, tantôt par la trop grande exigence de tel enseignant, tantôt par l'autorité d'un autre, ou simplement par leur manque d'intérêt, leur procrastination: «je n'aime pas les maths, c'est une punition»... Les équations mathématiques ne me serviront à rien», écrivait Latifa; «c'est parce que je ne travaille qu'à la dernière minute que j'échoue souvent», écrivait Mohamed dans son journal. Chez Sana, orpheline, la narration spontanée traitant de sa relation avec son père lui a permis un recul critique. En relatant leurs disputes et les contextes de celles-ci, elle a pu se rendre compte qu'elle avait, par «narcissisme», sa part dans le mainticn du conflit. Sa prise de conscience a apaisć sa vie familiale (d'après ses écrits) et a eu un effet observable sur sa vie d'élève: elle n'est plus enfermée sur elle-même, elle ne pleure plus en classe, elle s'investit de plus en plus dans les activités scolaires.

D'autres aspects relevant d'une réflexivité prospective, qui affleure à chaque retour sur l'expérience vécue, antérieure ou présente, sont à souligner. Les lacunes, les difficultés et les aspirations, une fois formalisées et verbalisées, impliquent l'élève dans une anticipation visant le dépassement des écueils et la concrétisation de ses objectifs. À titre d'exemple, questionnant sur le coup son incapacité à appliquer, lors des épreuves, les règles grammaticales étudiées en FLE (problème dit de trans-substantiation de la théorie et de la pratique, Weigand \& Hess 2007) - Meryem s'est proposée d'expliciter les règles morphosyntaxiques d'au moins deux phrases à chaque entrée de son journal. Dans la même perspective, d'autres élèves ont utilisé le journal comme support de préparation des exposés et des exercices, de consignation des fiches de lecture en vue d'éventuelles interventions en classe. Ils l'ont, ainsi, transformé en un atelier pour l'entraînement à la pensée et à l'écriture (Rosati 2013) où on voit apparaître aussi la dimension de tiers-éducatif du journal d'apprentissage (Pineau 2012). Libérés du maître, les élèves deviennent plus autonomes, ils apprennent librement à élaborer leur style et à s'acculturer en écrivant: ils empruntent des mots et des expressions aux supports ou à leurs lectures comme le faisait Safa, lectrice de G.K. Gibran et d'Aristote; ils explicitent les règles syntaxiques du français, en notant par exemple le temps auquel un verbe est conjugué; d'autres ont fait preuve de créativité en recourant au collage et dessin, comme Yassine qui, lorsqu'il lui manque un mot, le visualise par un dessin à rectifier par écrit une fois au collège.

Par ailleurs, le journal a eu des effets thérapeutiques émancipateurs, et a favorisé l'épanouissement de certains élèves. Pour 


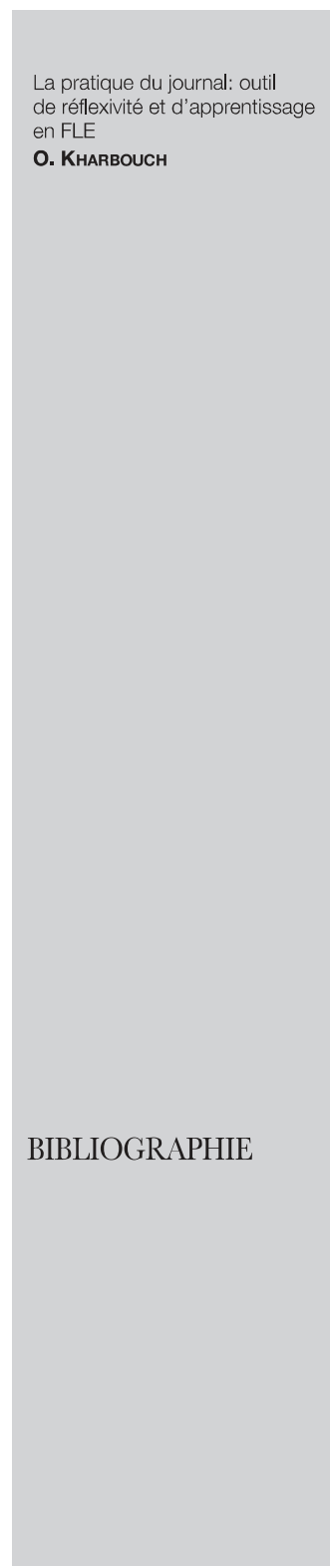

Rachid, par exemple, le journal a rempli la fonction de «squiggle» (gribouillis) psychanalytique (cf. Winnicott): sa relecture assistée a permis de diagnostiquer que c'est en étant conseillé (par une prof) de consulter un psychologue que Rachid s'est reconnu «psychopathe». Après ce diagnostic, il s'est imposé en classe en participant aux groupes et aux leçons. Quant à Safa, en lisant régulièrement des extraits de son journal en classe, elle a regagné progressivement confiance en elle. Elle s'est même investie auprès des classes du primaire de son village pour y animer des exposés; ce qui a étayé sa bonne réputation et lui a fait éviter d'être suspendue de l'école à la fin de l'année scolaire, comme la coutume l'exige aux élèves villagcoises de quinze ans.

Enfin, à partir de l'analyse de ces aspects, on voit que l'écriture permet aux élèves de déployer une réflexion sur le moins audible de leur vécu d'apprenants. Ils la déploient sous forme de critique spontanée adressée à soi-même ou bien à un autre, comme l'élève Anass l'a fait en écrivant à mon sujet: «c'est qu'il parle trop vite parfois et ne nous donne pas le temps pour le comprendre que je suis toujours obligé de demander à mon camarade ce qu'il a compris lui..». Ce qui, de fait, amène à souligner, en plus du rôle éducatif du journal, le rôle communicationnel de son «extimité» qui transforme les corpusjournaux en un espace de dialogue. Celui-ci accueillant un retour d'information (feedback), dont l'enseignant-lecteur pourrait se servir pour réinterroger la relation pédagogique, adapter sa pratique aux besoins des apprenants et interagir avec eux. Une «extimité» qui aurait pu enrichir et favoriser chez mes élèves une réflexivité collective et une «critique en permanence du savoir et des formes de la vie et de l'expériences (Rui 2010) s'ils avaient la possibilité de partager, dans notre milieu rural, leurs expériences par Internet.

CORMERY A. C. 2014. Le journal, outil d'analyse institutionnelle dans le travail social, entre intervention et militantisme. Thèse de doctorat en sciences de l'éducation. Université Paris 8.

HESS R. 2006. La pratique du journal, comme construction du moment interculturel. Paris: Universités UFR 8.

HESS R. 2016, 1. L'écriture du journal comme outil de formation de soi-même. Le Télémaque n43: 139-152.

HESS R. \& GRÉPEAU B. 2010 (octobre). Le journal de recherche. (B. Crépeau, Intervieweur)

KORCZAKJ. 2006. Les moments pédagogiques. Paris: Economica.

LAHIRE B. 2008. De la réflexivité dans la vie quotidienne: journal personnel, autobiographie et autres écritures de soi. Sociologie et 


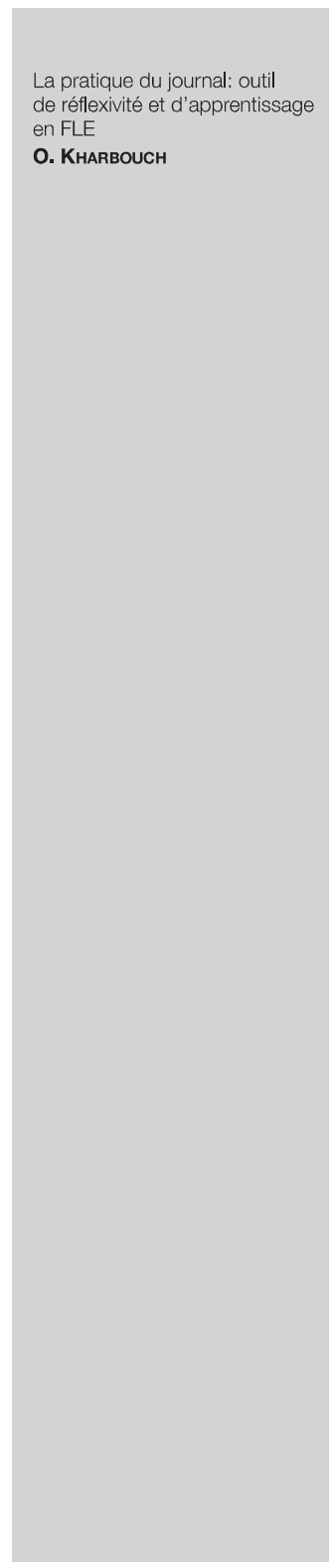

sociétés $\mathrm{N}^{\circ} 402$.

LAMIHI A. 1996. Janusz Korczak: L'éducation constitutionnelle. Descellée de Bouwer (Poche).

LITTLE D. \& (al). 2011. Le portfolio européen des langues: Rétrospective (1991-2011). Publication du conseil de l'Europe.

LOMBARD J. 1987. Malinowski Bronislaw, Journal d'ethnographe. Revue française de sociologie 28-2: 350-356.

LOURAU R. 1988. Le journal de recherche: Matériaux d'une théorie de l'implication. Paris: Méridiens Klincksieck.

PINEAU G. 2012 (Avril). Histoire de vie et formation de soi au cours de l'existence. Sociétés nº118: 39-47.

ROSATI M. V. 2013, 9. Écrire et écrit: le journal comme atelicr d'entraînement de la pensée. Pratiques de formation: analyses, $\mathrm{n}^{\circ} 62-$ 63: 89-95.

RUI S. \& (al), P. S. 2010. Réflexivité, Les 100 mots de la sociologie. Presses universitaires de France, coll. «Que Sais-Je?».

WEIGAND G., \& HESS R. 2007. La relation pédagogique. Anthropos. 